\title{
LA TRADUCCIÓN EN EL MANUAL SÁHARA: LIBRO DE LECTURA BILINGÜE PARA EGB \\ Larosi Haidar \\ Universidad de Granada
}

\begin{abstract}
In the former Spanish province number 53, and since the late sixties, the bilingualism became an evident linguistic phenomenon between Saharawi youth schooled. A clear proof of this bilingualism is the bilingual manual reading published in the midseventies, a book trying to reflect the new linguistic reality that was being forged in the streets of the Spanish Sahara. This work attempts to provide an overview of the manual and an introduction to its translational aspects.
\end{abstract}

KEY WORDS: translation, culture, bilingualism, Spanish Sahara.

\section{RESUMEN}

En la entonces provincia española número 53, y ya a finales de los años sesenta, el bilingüismo se convierte en fenómeno lingüístico palpable entre la juventud saharaui escolarizada. Una prueba manifiesta de este bilingüismo es el diseño de un manual bilingüe de lectura a mediados de los años setenta, manual que intentaba reflejar la nueva realidad lingüística que se estaba forjando en las calles del Sáhara Español. Este trabajo intenta ofrecer una descripción de dicho manual así como una introducción a los aspectos traductológicos que lo caracterizan.

PALABRAS CLAVE: traducción, cultura, bilingüismo, Sáhara español. 


\section{INTRODUCCIÓN}

Durante los últimos lustros de la pertenencia del Sáhara Occidental al Estado español, tiempos en los que era denominada la provincia número 53 y que era tan española como Cuenca o Guadalajara, los jóvenes saharauis empezaron a asistir con cierta asiduidad a las escuelas y centros formativos que la España franquista había ido fundando a cuentagotas en los núcleos urbanos más poblados. Tras décadas de reticencia y resistencia a cualquier tipo de inclusión en el sistema educativo metropolitano, finalmente las necesidades y los cambios radicales que estaban sucediendo en el sistema económico y social de la población indígena saharaui precipitaron la llegada masiva de estos nómadas del desierto a las pequeñas ciudades levantadas tímidamente en los últimos veinte años $\mathrm{y}$, por ende, la de sus hijos a las aulas agobiantes y claustrofóbicas de ladrillo y cemento.

Una de las primeras consecuencias de este acercamiento fue la inevitable interacción entre las dos comunidades españolas presentes en la provincia africana, es decir, por una parte los españoles hispanohablantes cristianos procedentes en su mayoría de las Islas Canarias y, por otra, los españoles hassanohablantes musulmanes en su mayoría autóctonos de la propia provincia del Sáhara Occidental. Esta interacción al ser diaria en escuelas, colegios y centros de formación va a tejer los cimientos de una sólida y pacífica convivencia que será la tónica general del entramado social vigente en el territorio hasta la muerte de Francisco Franco Bahamonde. Había nacido el germen de una sociedad española multicultural en la que cabían todos los integrantes de la sociedad civil de entonces, musulmanes y cristianos, hispanohablantes y hassanohablantes que, en pocos años, se convertirían en bilingües.

No es menos cierto que ya había un gran número de personas bilingües, sobre todo entre la población española saharaui que trabajaba en la administración pública o en empresas privadas provenientes de otras provincias, sin embargo, no es hasta finales de los años sesenta cuando realmente el bilingüismo se convierte en fenómeno lingüístico palpable entre la juventud saharaui escolarizada. Como el motor central de dicho bilingüismo va a venir accionado por las escuelas de educación primaria donde el programa docente normalizado es impartido en español, es evidente que la necesidad de adopción de una nueva herramienta de comunicación, en este caso la lengua española, la van a tener en exclusiva los estudiantes saharauis autóctonos cuya lengua materna es el hassanía. Ellos serán los bilingües por excelencia, sin olvidar que había un porcentaje no despreciable de jóvenes bilingües cuya lengua materna era el castellano. De hecho, hubo casos de un bilingüismo tan profundo y asimilado que desembocó en un biculturalismo arraigado y vivido 
con tanta intensidad que llevó a jóvenes españoles cristianos, originariamente de lengua materna castellana, a elegir el camino de la lucha armada al lado de sus hermanos y compañeros de aula saharauis, una vez que las autoridades españolas habían claudicado ante las presiones marroquíes y las Fuerzas Reales de Marruecos comenzaron la invasión del Sáhara Español, la quincuagésimo tercera provincia española.

Es en este contexto de convivencia cultural y lingüística y de un incipiente bilingüismo en el que nace la lógica necesidad de creación y obtención de material educativo bilingüe. Las dos lenguas en continuo contacto en el ámbito oral necesitaban hermanarse y reflejarse mutuamente en el ámbito escrito. Si ya la juventud saharaui española de aquellos años se había encargado de traducirse sus jergas habladas de una lengua hacia otra y hasta había logrado construir un híbrido hassanía-español tan natural y consolidado como los dos idiomas que lo conformaban, se echaba en falta, sin embargo, la plasmación de dicho hermanamiento lingüístico en la lengua escrita. Era evidente que el material educativo, en especial los libros de texto, de alguna manera cojeaba y padecía de un monolingüismo que día tras día parecía menos adecuado a la nueva realidad lingüística y cultural.

No sabemos si hubo más intentos de solventar este problema, lo que sí queda manifiesto es que el texto al que hemos tenido acceso es una prueba tangible de las preocupaciones e inquietudes educativas imperantes en el último periodo de la presencia española en el Sáhara Occidental. Había que confeccionar un libro de consulta bilingüe específico para los estudiantes de primaria de la provincia cincuenta y tres. Y alguien lo hizo.

\section{DESCRIPCIÓN DEL LIBRO}

No hemos podido acceder a un ejemplar original pues únicamente encontramos una copia del mismo facilitada por el dueño de una biblioteca personal. Está compuesto por 96 páginas sin numerar de $14 \times 21 \mathrm{~cm}$, precedidas * por una portada cuyo centro está ocupado por el título en tamaño grande y letras mayúsculas: SAHARA. Luego, viene el subtítulo, también en mayúsculas y letras más pequeñas: LIBRO DE LECTURA BILINGÜE PARA E.G.B. En la parte superior y centrado, figura en mayúsculas el nombre del autor: FERNANDO LUIS ALVAREZ CRESPO (sic.) seguido de "Maestro nacional". Centrado y en la parte inferior de la portada, aparecen los datos: "AAIUN-Sahara, 1975". Después, le sigue una segunda portada que conserva las inscripciones inferior y superior y cambia el título en español por uno en árabe escrito a mano, con erratas y caligrafía mediocre. Sin mediar índice ni introducción ni prólogo de ningún tipo, el lector se encuentra bruscamente 
inmerso en los diferentes textos y apartados que componen la singular monografía bilingüe $y$, como dice el título, los textos y apartados van apareciendo en parejas: el texto español y su traducción al árabe ${ }^{1}$.

Las diferentes lecturas propuestas en el libro son las siguientes:

Desierto: un bello poema que invoca al desierto y lo describe en todas sus contradicciones: "Eres como la flor [...]. Tierra quemada".

Cambio de vida en el Sahara: una breve descripción del duro hábitat saharaui en la que se resalta el vigor y la rudeza necesarios para sobrevivir en él.

Situación de Sabara en África (sic.): un mapa político de África que refleja la situación del Sáhara respecto al resto de territorios africanos. Las denominaciones que aparecen en el mapa son Islas Canarias, Marruecos, Sáhara, Argelia y Mauritania.

Sahara: mapa aislado del Sáhara Occidental en el que aparecen puntos numerados correspondientes a localidades. Una leyenda ubicada a la derecha del mapa lo confirma presentando una lista de veintiuna localidades saharauis. En la parte inferior, se nos presenta un cuadro con "Datos de interés" que incluyen superficie, población, temperatura media, altitud máxima, clima, distancia de la capital Madrid, longitud de la costa y la longitud de la frontera terrestre. En las siguientes dos páginas, hay una sucesión de puntos apartados que se presentan como títulos en mayúsculas y que más bien sirven de desarrollo resumido de los anteriores citados en el apartado Sahara. Cada uno va acompañado de una pequeña explicación que no se extiende más allá de unas cuantas líneas.

Estos apartados son: SITUACIÓN, SUPERFICIE, LÍMITES, COSTAS, HIDROGRAFÍ, CLIMA, FLORA, FAUNA, LENGUA, POBLACIÓN, RELIGIÓN, DENSIDAD DE POBLACIÓN (sic.). Tras el último apartado, aparece un bosquejo a mano de un paisaje de dunas.

Orografía: presenta un pequeño resumen del relieve saharaui y su descripción, además de un mapa físico del Sáhara Occidental que sitúa las zonas geográficas más destacadas del territorio. Le siguen varios apartados que aumentan la información ofrecida anteriormente: Hidrografía, Costas y Clima.

Agricultura: en este apartado se ofrece un resumen sobre lo que hay que saber de las pocas verduras y otros cultivos que se dan en el Sáhara, así como de las dificultades naturales que un medio como el desierto suele presentar ante la práctica de la agricultura en general.

1 En realidad, aunque la traducción en líneas generales intenta respetar la estructura y la morfosintaxis del árabe estándar, lo que se obtiene finalmente es un texto árabe salpicado de hassanismos y usos locales que más bien afean y entorpecen la lectura y comprensión. Sin contar, claro está, los errores, las erratas y las anomalías fruto -o esa es al menos nuestra conclusión- de la posición de inferioridad adoptada por el traductor frente al texto castellano traducido. 
Ganadería: breve presentación del ganado saharaui en la que la estrella, obviamente, es el camello. El texto acaba con una frase muy curiosa: "El cebú, animal que se adapta perfectamente al desierto, puede ser el ganado del futuro del Sahara por su rusticidad y gran rendimiento de carne".

Pesca: apartado que destaca la importancia de la fauna marina de las costas saharauis. Una imagen que apoya al texto nos presenta a un pescador en traje de buzo luciendo entre sus manos un enorme ejemplar pescado en las aguas de la provincia africana.

Minería: como no podía ser menos, en este punto se destaca la importancia de los yacimientos de fosfatos de Bu-Craa. Además, el apartado lo encabeza un párrafo que, de alguna manera, podría explicar y despejar muchas dudas que hoy en día parecen enmarañar el intrincado problema del Sáhara Occidental ocupado por Marruecos:

Los geólogos han previsto formalmente la posibilidad de que en el Sahara existen yacimientos de petróleo. La zona sedimentaria en la que pudieran hallarse los hidrocarburos ocupa una enorme extensión.

Tras esta pequeña introducción a la minería y a los fosfatos de Bu-Craa, sigue una especie de pequeño estudio más exhaustivo del tan querido y codiciado yacimiento. El autor lo divide en varios apartados en los que desglosa los pormenores de todo el proceso de explotación de los fosfatos, desde su extracción del corazón del desierto, en Bu-Craa, hasta su carga en grandes buques realizada casi cien kilómetros más al oeste, en la playa de Aaiún. El título del estudio es Yacimiento de Bu-Craa y los apartados que lo componen son: Situación, Reserva, El fosfato, La explotación, Cinta transportadora y Cargadero. El apartado dedicado a la cinta transportadora incluye una tabla de datos en la que se habla de las características principales de la cinta (longitud total, anchura de la banda, número de tramos, desnivel, etc.). Una foto del cargadero, con el muelle al fondo y una grúa en plena actividad, introduce el último apartado del pequeño estudio.

Fauna del Sahara: introducción algo más extensa a los animales salvajes que pueblan el desierto saharaui. El apartado lo encabeza la imagen de un fenec que el autor define de la siguiente manera: "Muy curioso es el fenec, de grandes orejas, vivísimos ojos y agilísimos movimientos. Los saharauis lo llaman 'zaalab"'. Dentro de este apartado hay varias entradas correspondientes a animales característicos del territorio saharaui y que se ilustran con imágenes de los mismos hechas a mano: Guepardo, Jerbo, Gacela y Tarro canelo.

Flora del Sáhara: aunque en este apartado se enumeran unas cuantas plantas típicas del Sáhara Occidental, en realidad es la acacia la que recibe toda la atención del texto y, como dice el autor, esta acacia, "la 'talha', para el 
hombre del desierto, es como un maná. Le sirve como punto de referencia [...]. Su sombra es refugio para los animales e incluso para él". Un dibujo a mano de la planta más famosa del desierto le sirve de fondo a toda la página que ocupa este apartado.

Aspectos de la vida saharaui: en la práctica, es una escueta información sobre las prácticas religiosas saharauis. Las últimas líneas dicen así:

El saharaui es profundamente religioso y manifiesta exteriormente su religiosidad de forma permanente. Son frecuentes, así mismo, las peregrinaciones a tumbas de santones famosos.

Organización Social: una foto de lo que parece un aula de algún colegio introduce el apartado. Las personas que ocupan los pupitres son mayores de edad. El breve texto se limita a nombrar algunas de las tribus saharauis más destacadas, destacando el aspecto tribal y patriarcal de la sociedad saharaui:

La autoridad del padre o el hermano mayor es indiscutible y de por vida $\mathrm{y}$ tiene su fundamento en un principio moral: los mayores son depositarios de la tradición, de la prudencia, de la sabiduría y de la experiencia.

Distintivo Saharauis: en este apartado todo está escrito a mano y es una representación de los símbolos y letras que sirven de distintivo a las diferentes tribus y agrupaciones tribales del Sáhara Occidental.

Yemaa General del Sáhara: tras una fotografía de la Yemaa en plena sesión, aparece un texto que nos desglosa los diferentes órganos que forman la Asamblea General del Sáhara.

Vestimenta Saharaui: una presentación escueta de las diferentes prendas que suelen vestir los habitantes del Sáhara Occidental. En primer lugar, aparece la lista de las prendas masculinas y, después, la de las prendas femeninas que el autor remata con la siguiente frase: "Las palmas de las manos y plantas de los pies suelen pintarlas de color ocre y las uñas al rojo vivo (sic.)". Para complementar este apartado, le siguen otros dos que se limitan a presentar un pequeño glosario que incluye prendas y otros términos: Vestidos y Varios.

Vivienda: la mitad inferior de la página la ocupa un dibujo a mano de la típica localidad árabe del desierto que los orientalistas se han encargado de difundir por todos los rincones del planeta: palmeras inclinadas eternamente, dunas en repetición matemática $\mathrm{y}$, al fondo, casuchas medio invisibles atravesadas amenazadoramente por la imponente torre minarete de la imprescindible mezquita. En la parte superior, el texto hace hincapié en la jaima como vivienda y hogar de los habitantes del desierto. En las páginas siguientes, 
otros cuatro glosarios bilingües se suman a los anteriores: La Casa, Meses del año, La Clase y El Saludo.

Cocina Típica: en este apartado se describen brevemente varios platos de los más conocidos y servidos en las mesas saharauis. También se habla del té y de la archiconocida generosidad del saharaui:

El saharaui es generoso [...]. Una boda, un bautizo, la circuncisión de un niño o la simple visita de un familiar o de un amigo son motivos más que suficientes para dar una fiesta.

Folklore: lo que más bien se intenta describir bajo este título es, de manera exclusiva, la danza de la mujer saharaui. Un dibujo de un tambor ilustra el breve texto.

Artesanía: un dibujo que intenta en vano presentarnos la cara de una mujer saharaui, pues más lo consigue puesto al revés, le sirve de apoyo a un texto corto que empieza así:

La artesanía tradicional es una actividad que está localizada exclusivamente en los 'majaderos', artesanos que practican varios oficios, todos ellos en forma primitiva y elemental.

El Té: este es uno de los apartados más exhaustivos y en el que se distinguen tres partes claramente diferenciadas. En una primera parte, se realiza una introducción sobre la planta del té, su historia y su introducción en Occidente. Ilustraciones a mano representando las hojas de té, cajetillas y bolsitas, le sirven de fondo al texto escrito. La segunda parte, adornada con dibujos de algunos utensilios, nos sumerge en la ceremonia del té. El autor la culmina como sigue:

Se toman tres vasos de té: el primero se relaciona con la amargura de la vida; el segundo con el estío (sic.) de la juventud, y el tercero con la vejez. Fuerte, suave y dulce, así son los tres vasos de té.

La tercera parte, titulada Elementos del té, consiste en un glosario que recoge las denominaciones de los utensilios de té más habituales. Más dibujos de otros tantos utensilios ilustran el texto escrito.

La Jaima: una primera ilustración parece más bien presentarnos una tienda playera que una típica jaima saharaui. El texto, relativamente largo en comparación con el resto de apartados, ofrece información relativa a varios aspectos de la jaima. En la segunda página, el dibujo que ilustra el texto se acerca más a lo tradicionalmente conocido como jaima saharaui y, finalmente, un glosario con varios términos referentes a la vivienda del desierto. 
Una escena curiosa y siempre repetida es la que se ofrece al viajero al llegar a un 'frig'. Al hacer alto en su camino, mientras 'barracan' los camellos, verá avanzar hacia él un hombre curtido que con aire majestuoso acude a darle la bienvenida. [...]. Se ha dicho, y con razón, que la jaima es la nave del desierto.

Tribunales de Kodat: una escueta introducción a la justicia en uso en la sociedad saharaui: "El Tribunal de Kodat trata de sacar a la luz, la solución de aquellos casos que fueron fallados en primera instancia por el Cadí local correspondiente". Apoya el texto el dibujo de dos hombres saharauis con su vestimenta tradicional y sentados en el suelo mientras supuestamente discuten sobre algún asunto.

Léxico: un pequeño glosario bilingüe formado por términos sobre alimentación y vivienda.

Imposición del nombre: dos dibujos parecen representar a un hombre y un niño saharauis en ropa tradicional. El texto escrito ofrece una breve descripción de la ceremonia en la que se le designa nombre al recién nacido:

El día que se da nombre al recién nacido, se sacrifican varias cabras y ovejas. Se invita a todos los familiares y vecinos para festejar el bautizo. Las fiestas duran tres días. En ellas se baila, se tocan palmas al son del tambor y se toma el té en gran cantidad, mientras los familiares obsequian con regalos al recién nacido. La fiesta termina dando gracias a Alá por el nuevo miembro de la familia.

Boda saharaui: escueta y singular descripción de la ceremonia del matrimonio, desde los preparativos iniciales hasta que los novios se van a vivir solos.

Los festejos de la boda suelen durar tres o sietes días, según la economía del novio. Todos los alimentos han de ser comprados por él y hechos en la jaima del padre de ella. Por las tardes se reúnen los jóvenes para cantar y bailar.

Calendario musulmán: último capítulo del libro que recoge una definición del calendario lunar y la notación de los meses en su denominación árabe más una pequeña explicación de las actividades que suelen hacerse en cada mes.

MOHARREN, mes sagrado del Año Nuevo, 30 días.

SAFAR, mes de partida para la guerra, 29 días. 
Una vez realizada esta pequeña descripción introductoria de este manual bilingüe que debió ser un texto pionero en aquellos últimos años de la presencia española en su última provincia del continente africano, pasamos al análisis traductológico de las dos componentes lingüísticas del mismo, es decir, un estudio comparativo de los textos españoles y sus equivalentes árabes.

\section{SOBRE LA TRADUCCIÓN}

En este acercamiento al manual, nos limitaremos a destacar los aspectos más llamativos que de alguna manera definen su esencia bilingüe y, por lo tanto, su vertebración sobre dos textos de los que uno es traducción del otro. Un estudio más exhaustivo que abarque todos los capítulos y sus traducciones, y que desgrane una a una las diferentes opciones de traducción y soluciones adoptadas por el traductor, necesitaría mucho más espacio y dedicación que la permitida en el presente formato.

La primera observación que cabría realizar es que no figura en ninguna parte cuál es el texto original y cuál el texto traducción. Igualmente, tampoco aparece el nombre del traductor. Únicamente, aparece nombrado el autor, Fernando Luís Álvarez Crespo "maestro nacional”. Podría ser que el autor sea a la vez el traductor, en cuyo caso probablemente habría considerado innecesario citarse a sí mismo como traductor. Por nuestra parte, únicamente podremos inferir ciertas características del autor de los textos partiendo de la esencia misma de estos textos y de su vinculación morfológica y semántica.

El primer rasgo que cabría destacar es que ciertas partes del texto árabe están escritas a mano, concretamente los títulos y subtítulos, inclusive los de la portada. La caligrafía está poco cuidada y no parece que el amanuense en cuestión se haya esmerado mucho en lograr presentarnos una letra en caracteres árabes más o menos aceptable desde el punto de vista estético. Pero tampoco podemos felicitarle por la corrección ortográfica, pues en muchos casos los errores son garrafales y no parece que todos puedan ser considerados como simples erratas. Este sería el caso del título del libro, que aparece como sigue:

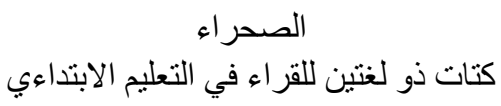

Si tenemos en cuenta que la escritura ha sido realizada a mano y que las palabras utilizadas son bastante corrientes y de gran difusión entre los hablantes de árabe con un mínimo nivel de estudios, la hipótesis de las erratas se hace insostenible para este caso. La palabra kität (كتات) salta a la vista y llama la 
atención de cualquier lector árabe, de allí nuestra consideración de que es más bien un error. La palabra correcta sería kitāb (كتاب) y es difícil saber el origen de este error a excepción de algún tipo de confusión entre las dos letras finales de las dos palabras. La otra palabra en la que figura un error es la última, al-ibtidā(الابتداءي), en la que la hamza (s) aparece independiente en vez de estar sobre la $i \bar{a}$ (ي) que es lo correcto. Si el texto estuviera escrito a máquina u ordenador, se podría achacar la falta a un fallo a la hora de teclear la palabra, sin embargo, al estar escrito a mano no hay duda de que ha sido un error.

Siempre en el ámbito del título, podemos observar más anomalías vinculadas esta vez a la traducción. Al término castellano bilingüe le corresponde en árabe ذو لغتين, es decir, “de dos lenguas”, que evidentemente no es una expresión muy adecuada al contexto ni puede ser calificada de natural y usada convenientemente en la frase. Consideramos que este uso forzado de la expresión "de dos lenguas" es más bien debido a que el traductor opta por ceñirse a las palabras y expresiones según surgen en castellano, lo que ya de por sí nos está aportando una información muy relevante: el texto castellano es el texto origen y el texto árabe es su traducción. De hecho, si nos atenemos a la corrección lingüística y al cuidado de la caligrafía, es obvio que el título en mayúsculas, el título verdadero y serio, el título que manda, es el que está plasmado en castellano; mientras que el otro, el escrito de cualquier manera, el que más bien está garabateado, el que se sacrifica por imitar la forma del primero, es el título expresado en árabe, el título subordinado.

Una vez más, parece que la esencia de la traducción, la esencia del texto resultante de traducir otro texto primigenio, es, ante todo, una esencia subordinada y ligada al azar morfosintáctico de la lengua traducida. De hecho, este es un fenómeno que se observa en muchas traducciones realizadas por traductores bisoños o sin formación alguna, traductores para quienes la traducción es una camisa de fuerza que se nos impone y ante la cual nada podemos hacer que no sea dar gritos y patadas a diestro y siniestro, y que el otro, el lector, entienda lo que pueda. Y sin embargo, la traducción es comunicación, es comprensión. Esto es, naturalidad, cohesión y coherencia. Es expresar en nuestra lengua lo que dice el otro en la suya, respetando sus objetivos e intenciones y teniendo en cuenta los contextos socioculturales que, precisamente gracias a esta traducción, están interactuando.

Después, aparece un mapa de África en el que se recogen las divisiones y fronteras, fruto del colonialismo, adoptadas por la mayoría de las naciones africanas una vez independientes. En todo el mapa, o más bien croquis, que incluye toda África, Oriente Próximo y los países de Europa meridional, sólo aparecen mencionados literalmente el Océano Índico, el Océano Atlántico, las Islas Canarias, el Sáhara [Español], Mauritania, Marruecos y Argelia. Sin embargo, el traductor en este caso no traduce los nombres como cabría esperar 
sino que opta por conservar en las dos versiones las denominaciones en castellano. Únicamente traduce la notación que encabeza el mapa:

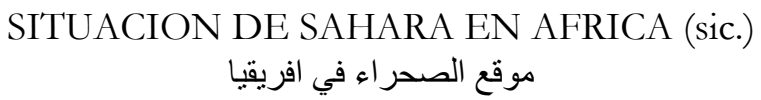

La causa de esta opción no podemos saberla con seguridad, suponiendo que haya sido una opción pues podría haber sido fruto de un despiste del traductor.

Pasamos a ver un segundo mapa, en este caso del Sáhara Occidental, en el que aparecen veintiún puntos numerados del (1) al (21). Se trata de las principales localidades saharauis que se encuentran dispersas por todo el territorio de la entonces quincuagésimo tercera provincia española. En este caso, el paralelismo entre los dos textos es notable: los títulos Sahara (sic.) y , تعليمات ذات أهمية y finalmente las dos listas de localidades numeradas que acompañan a sendos mapas de la provincia africana. Aquí cabría destacar un error de traducción evidente que es el hecho de traducir en el contexto que nos ocupa la palabra datos por تعليمات, es decir, instrucciones, lo que podría deberse a desconocimiento terminológico o a confusión fonética, pues ta'limāt² podría confundirse fácilmente con ma'lümàt ${ }^{2}$.

En este mismo mapa, concretamente en la lista numerada de localidades saharauis, hay dos denominaciones que han llamado nuestra atención y que posiblemente nos ofrezcan pistas sobre la identidad del traductor. Por una parte, está el nombre árabe que corresponde al español Cabo Bojador y ocupa el número cuatro de la lista, nombre que ningún buen conocedor de la cultura saharaui utilizaría. La denominación saharaui, supuestamente la primera y de la que partió el nombre europeo 4 que intenta imitarla fonéticamente, siempre ha sido Būydūr (بوجدور), elidiendo sistemáticamente la referencia al accidente geográfico que alberga la localidad. Es verdad que existía también otra transcripción como la usada por Edward Bold en su The Merchants' and Mariners' African guides. Dice así en la página once: "From Cape Bogador to Cape Blanco the course is about S.S.W.1/2 W. 125

${ }^{2}$ Transcripción fonética de la palabra árabe utilizada por el traductor y que significa instrucciones.

${ }^{3}$ Transcripción fonética de la palabra árabe que serviría como traducción de la castellana datos.

${ }_{4} \mathrm{Al}$ parecer, existe la posibilidad de que la denominación europea, concretamente portuguesa, no sea un intento de transcribir la denominación saharaui sino más bien una alusión al aumento de la actividad remera de los marinos al acercarse a la región del cabo. El término bojador provendría de bojar, "rodear navegando una isla, cabo o porción saliente de la costa". Además, a veces el cabo viene anotado como Bogador, que provendría de bogar, es decir, remar. En portugués, bojar sería "maniobrar un navío recorriendo la costa".

5Edward Bold (1822). The Merchants' and Mariners' African guide. The coast, bays, barbours, and adjacent islands of West Africa. Nueva York: Cambridge University Press. 
leagues, as far as Angra dos Ruivos”. Sin olvidar que más al norte, esta vez en territorio marroquí, existe otro cabo denominado Mogador y cuya denominación difícilmente puede considerarse de origen europeo. Por analogía, si Bogador (Bojador) es en árabe Būydūr, es decir, "el que tiene muchos aydūr" entonces Mogador provendría del árabe amaydūr, esto es, "la que tiene muchos aydür'. También es bueno saber que a la misma altura de Mogador y a unos pocos kilómetros hacia el interior se encuentra una pequeña localidad marroquí denominada Ain Oum Jdour, que vendría a significar "manantial de la que tiene muchos aydūr". Si añadimos que yudūr (جدور) aparece en el diccionario árabe Almunyid' como plural de yadr y yidr, cuyo significado es "planta que crece en la arena", parece más que probable el origen local de la denominación pues, al menos en el caso de Bojador, existe abundante vegetación que responde a la citada clasificación. Una etimología del término verdaderamente extraña es la pretendida por Adriana Kreiman: "El nombre Bojador aparece bajo diversas formas: Bu Jaydur, Bujdur, Boujdour, Bojador, Cabo Bojador. Su nombre en árabe, 'Abu Jatar', significa 'el padre del peligro"'7.

Sin embargo, lo que sí es cierto es que un saharaui hablando en hassanía, y menos todavía en aquellos años de mediados de los setenta del siglo veinte, jamás dirá que tiene la intención de viajar a Cabo Bojador sino, simplemente, a Bojador. En este sentido, podemos descartar sin miedo a equivocarnos, que el texto origen haya sido el texto árabe o, al menos, en lo que respecta a la denominación de Cabo Bojador la lengua de la que se ha traducido ha sido el español. Además, sería raro que el traductor de este término al árabe sea un gran conocedor de la toponimia saharaui y su idiosincrasia, y ello por la argumentación anteriormente mencionada. Sin olvidar la verdad de que muchos traductores se convierten en esclavos de la sintaxis del texto original y aun siendo buenos conocedores de la terminología y usos lingüísticos del idioma al que traducen acaban poniéndose ellos mismos una camisa de fuerza que los inmoviliza y desnaturaliza sus textos.

Por otra parte, está la notación número quince de la lista, que aparece en castellano como BirNzaran, obviamente transcripción de la denominación árabe hassaní. Sin embargo, el equivalente de la lista numerada árabe es $B \bar{r}$ - $A n$ naśrāni (بير النصراني), lo que no sólo se aleja fonéticamente de la denominación hassaní utilizada por los habitantes autóctonos sino que, además, su significado es totalmente erróneo y de ninguna manera se puede identificar

${ }^{6}$ Almunyid (1992). Beirut: Dar El MachreqSarl Publishers.

${ }^{7} \mathrm{En} \quad$ http://sobremarruecos.com/2010/03/12/bojador-donde-la-leyenda-se-vuelve-historia/ (22/06/2014). Suponemos que este dato habría sido recogido de Wikipedia, en la entrada dedicada a Enrique El Navegante, donde aparece dicha denominación en la versión árabe, pues es el único lugar encontrado que utiliza esta notación. 
semánticamente con el nombre saharaui de la localidad a la que hace referencia, es decir, Bìr-Anzarān (بير انزران).

Es bien sabido que gran parte de los topónimos todavía hoy utilizados en el territorio del Sáhara Occidental son de procedencia bereber, es decir, amarigh, y en el caso que nos ocupa dicha procedencia es indiscutible. La denominación hasaní está compuesta por un término árabe, Bīr, que significa pozo, y un término amazigh, Anzarān, plural de anzar, que significaría lluvia. Además, en la mitología bereber $A$ zar era el dios de la lluvia. De esta forma, el nombre de la localidad vendría dado por el del pozo, es decir, significaría "el pozo de las lluvias" debido a su importante caudal. Buchar Haidar lo cita como un "antiguo pozo muy importante y conocido situado al noroeste de la depresión de Nagyir en una bajura en la que desembocan todas las aguas torrenciales. Su agua es potable y abundante" .

Dicho esto, la denominación ofrecida por el autor como nombre saharaui de la localidad sería como mínimo inusual y poco conocida entre los

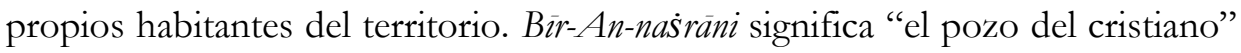
y que a primera vista cuesta relacionarlo con la transcripción hecha al castellano del nombre original hassaní. Sin embargo, si nos centramos en el segundo término de la denominación ofrecida por el autor en la lista española de localidades, esto es, Bir Nzaran, nos cercioramos de que leído en castellano apenas se diferencia de nazaran y nazareno, que es el étimo del árabe naśrāni o cristiano. En definitiva, es posible que haya habido una identificación errónea de Nzaran con nazareno y de allí su fallida traducción. Una vez más, queda claro que los caminos de la traducción y retraducción, de la transcripción y retranscripción son inescrutables.

\section{CONCLUSIONES}

Del acercamiento al manual, se desprende que la esencia de la traducción, la esencia del texto resultante de traducir otro texto primigenio, es, ante todo, una esencia subordinada y ligada al azar morfosintáctico de la lengua traducida. De hecho, este es un fenómeno que se observa en muchas traducciones que, generalmente, han sido realizadas por traductores para quienes la traducción es una camisa de fuerza que se nos impone y ante la cual nada podemos hacer que no sea dar gritos y patadas a diestro y siniestro, y que el otro, el lector, entienda lo que pueda. En el caso que nos ocupa y con apenas

8 Aparece como nombre masculino definido de la siguiente manera: “Anzar. (Norte de África). Significa lluvia y además hace alusión al nombre que según la tradición amazighe continental se le daría al dios de la lluvia" en http://izuran.blogspot.com.es/p/nombres-personales.html. 'Buchar Haidar (2012). Geografía del Sáhara Occidental. Madrid: Bubok (Edición en árabe, p.69). 
un par de ejemplos vistos, dicha subordinación ha quedado más que clara y la indiferencia hacia la lengua de traducción ha sido la tónica. Por el momento, el subalterno spivakiano (Spivak, 1988) sigue sin poder hablar.

\section{BIBLIOGRAFÍA}

Álvarez Crespo, F. (1975). Sáhara. Libro de lectura bilingüe para E.G.B. Aaiún, Editorial Gráficas Sahariana, Servicio de Publicaciones del Gobierno General del Sáhara.

Bold, E. (1822). The Merchants' and Mariners' African guide. The coast, bays, harbours, and adjacent islands of West Africa. Nueva York, Cambridge University Press.

Dar El Machreq (1992). Almunyid. Beirut, Dar El Machreq Sarl Publishers.

Haidar, Buchar (2012). Geografía del Sáhara Occidental. Madrid, Bubok.

Spivak, Gayatri (1988). "Can the subaltern speak?” en Nelson, C. y Grossberg, L. (eds.): Marxism and the Interpretation of Culture. Basingstoke, Macmillan Education, pp. 271-313.

Wikipedia [En línea]:

http://ar.wikipedia.org/wiki/\%D $\% \% 87 \% \mathrm{D} \% \% 86 \% \mathrm{D} 8 \% \mathrm{~B} 1 \% \mathrm{D} \% \% 8 \mathrm{~A} \% \mathrm{D} 8 \%$ A7\%D $\% \% 84 \%$ D8\%A8\%D8\%AD $\%$ D 8\%A7\%D8\%B1 [Consulta: $22 / 06 / 2014$ ] 